Trindade, I. A., Ferreira, C., Moura-Ramos, M., \& Pinto-Gouveia, J. (2017). An 18month study of the effects of IBD symptomatology and emotion regulation on depressed mood. International Journal of Colorectal Disease, 32(5), 651-660. doi: $10.1007 / \mathrm{s} 00384-017-2774-\mathrm{z}$

The final publication is available at Springer via

https://link.springer.com/article/10.1007/s00384-017-2774-z 
An 18-month study of the effects of IBD symptomatology and emotion regulation on depressed mood

\author{
Inês A. Trindade, MSc, PhD student ${ }^{1} *$ \\ Cláudia Ferreira, $\mathrm{PhD}^{1}$ \\ Mariana Moura-Ramos, $\mathrm{PhD}{ }^{1}$ \\ José Pinto-Gouveia, $\mathrm{MD}, \mathrm{PhD}^{1}$
}

${ }^{1}$ CINEICC - Cognitive and Behavioural Centre for Research and Intervention

Faculty of Psychology and Education Sciences

University of Coimbra, Portugal

*Corresponding author.

Inês A. Trindade, Faculdade de Psicologia e de Ciências da Educação da Universidade de Coimbra, Rua do Colégio Novo, 3000-115 Coimbra, Portugal.

Email: ines.almeidatrindade@gmail.com 


\title{
An 18-month study of the effects of IBD symptomatology and emotion regulation on depressed mood
}

\begin{abstract}
Depressive symptomatology in IBD patients is known to predict disease activity, which in turn can increase depressive symptoms in a perpetuating a cycle between depression and IBD symptomatology. The mechanisms that contribute to the relationship between disease activity and depressive symptoms are not clearly investigated yet. Since emotion regulation has been considered particularly relevant to define the impact of adverse experiences on different outcomes, the current study aimed to examine the longitudinal influence of two maladaptive emotion regulation processes, cognitive fusion and brooding, on the association between disease activity and depressed mood.
\end{abstract}

This study was conducted over an 18-month period, using a sample of 116 IBD patients that completed self-report validated measures in three different waves. Correlation analyses and cross-lagged panel models were performed.

The main result from this study discovered that the experience of IBD symptomatology at baseline, although positively linked to the manifestation of depressed mood 18 months later $(r=0.25 ; p<0.01)$, does not directly predict depressive symptoms. This relationship is rather indirect, as it is explained by the engagement in cognitive fusion $(p=0.028)$ and brooding $(p=0.017)$. These maladaptive emotion regulation processes, that were revealed to be consistent over time, link IBD symptoms with subsequent depressed mood.

These findings indicate that clinicians should be aware of the emotion regulation processes patients tend to use to handle difficult experiences. The inclusion of 
psychological assessments and interventions in the healthcare of IBD patients should be seriously considered. Further implications are discussed.

Keywords: depressive symptomatology; emotion regulation; cognitive fusion; brooding; longitudinal data. 


\section{Introduction}

\section{Inflammatory Bowel Disease and psychological distress}

Extensive research has highlighted the major impact that IBD holds on patients' quality of life (QoL) and psychosocial functioning [e.g., 1-3]. IBD often leads to feelings of shame, embarrassment and isolation, concerns regarding the risk of relapsing or developing cancer, difficulty in maintaining daily routines due to severe symptomatology that may arise abruptly, and struggles with body dissatisfaction and sexuality [4-8]. It has been reported that, even during periods of remission, IBD patients' QoL is significantly poorer in comparison with healthy controls, and that comorbid psychopathology is frequent $[1,9,10]$. In particular, results from a recent study that used structured psychiatric diagnostic interviews, showed that participants with IBD presented a rate of $27 \%$ of depressive disorder, compared to the $12 \%$ rate presented by a matched sample of controls with similar demographic characteristics [11]. IBD patients also seem to present higher levels of depression than patients with other chronic illnesses such as colorectal cancer [12].

Depressed mood in IBD is of special relevant given its association with disease activity. Longitudinal studies have found that depressed mood and associated anxiety may influence the disease course by predicting active disease and more relapses [13, 14]. This link might be explained by the relationship between depression and cellular and humoral immunity alterations [15]. In fact, it has been found that depressed mood may stimulate the production of proinflammatory cytokines $[16,17]$, modulating the clinical expression of IBD [6]. What is more, since increased IBD activity can influence the intensity of depression and anxiety experienced by patients [18-20], these mechanisms seem to unfold into a self-perpetuating cycle of psychological suffering and inflammation. It has thus 
been pointed out that more priority to this subject should be given, and that more efforts should be made to uncover the mechanisms associated with the known effect of IBD symptomatology on depression [21].

\section{Emotion regulation}

It is considered that other factors other than the direct illness manifestations and limitations may influence patients' well-being and quality of life [22]. Namely, psychological distress seems to mainly result from the way one deals with difficult experiences (e.g., adverse symptomatology, unwanted thoughts or emotions), rather than resulting from the direct impact of those experiences [e.g., 23, 24]. Individuals use different emotion regulation processes to handle adverse experiences; these processes are complex strategies that aim to modify the type, frequency, magnitude, or duration of internal experiences $[25,26]$. Maladaptive emotion regulation processes, which often involve tendencies to control, avoid, or get entangled with those experiences, present paradoxical effects and are linked to lower mental and physical health, predicting many forms of psychopathology, including depression (e.g., [23, 27]).

Cognitive fusion is a maladaptive regulation process that involves the dominance of cognitive events in one's experience and behaviour. This process involves the tendency to consider thoughts literal and believable interpretations of reality, and the inability to view thoughts as transitory and automatic mental events [23, 28]. Cognitive fusion thus refers to "the relationship a person has with this or her own cognitive events, on a continuum from fused (dominated by, entangled, believed, taken literally) to defused (experienced as mental events and not necessarily to be acted upon)" [28]. IBD patients, when "fused" with their cognitions, may get entangled with illness-related thoughts (e.g., "these symptoms are never going away"; "my illness is too embarrassing for me to talk 
about it with others"; "nobody will understand how this illness affects my life") [29] and consequently choose to behave as if they were literally true [30].

Rumination is another maladaptive regulation process that refers to the way a person handles aversive internal events. This process is characterized by self-reflection and repetitive and passive focus on one's negative emotions and possible causes and consequences [31-33]. Rumination does not lead to active problem solving and instead often immobilizes the person, predicting the onset, severity, and maintenance of depressive symptoms $[31,32]$. Brooding represents the most depressogenic form of rumination, being defined as "a passive comparison of one's current situation with some unachieved standard" [34], and comprising a focus on "why me?" issues and on the obstacles to overcome problems [33].

In particular, data regarding the role of cognitive fusion and brooding in IBD, although limited, has shown the pernicious impact of these processes on patients' reported depressive symptomatology [29]. Nevertheless, the one study conducted to date regarding the influence of these processes on IBD presents a cross-sectional design.

\section{Aims of the current study}

The present study aims to explore whether cognitive fusion and brooding influence the relationship between reported IBD symptomatology and depressed mood, using an 18-month longitudinal design. This study thus intends to determine whether these maladaptive emotion regulation processes are mediators of that relationship. We hypothesize that cognitive fusion and brooding will intensify the impact of IBD symptomatology on subsequent reported depressed mood.

\section{Material and Methods}




\section{Setting and participants}

This study is based on the longitudinal data from IBD patients recruited through the Portuguese Association for IBD (APDI), which invited its members registered as patients to collaborate in the study. Respondents were informed about their right to abandon the study at any time, about the procedures and voluntary nature of the study, and about the purpose and confidentiality of the data. All participants gave their informed consent.

Participants were asked to take part in three waves of assessment on an online survey, equally spaced approximately 9 months apart. Data collection thus lasted for one year and a half, from the end of 2014 to the beginning of 2016.

Wave 1 was completed by 209 participants, Wave 2 (9-month assessment) by 168 , and Wave 3 (18-month assessment) by 127 . Only the respondents who completed the three waves $(N=123)$ were considered for the present study. Of these participants, 7 were excluded for reporting other severe illnesses (breast cancer, fibromyalgia, thalassemia, multiple sclerosis, diabetes), or psychiatric conditions (bipolar disorder, generalized anxiety disorder).

This paper's sample thus includes 116 IBD patients (35 males and 81 females), comprising 70 patients with Crohn's Disease, 43 with Ulcerative Colitis, and three with IBD-unknown.

\section{Measures}

The research protocol included self-report demographic and medical history questions. Medical information comprised form of IBD, time since IBD diagnosis, frequency of IBD symptomatology during the previous month (10 symptoms measured 
on a 6-point scale), presence of associated medical complications, and number of undergone surgeries. Additionally, participants completed the Portuguese validated versions of the following instruments at the three waves of assessment:

Cognitive Fusion Questionnaire-7 (CFQ-7 [28]; Portuguese version by PintoGouveia, Dinis, Gregório, \& Pinto, 2014). The CFQ-7 comprises 7 items rated on a 7point scale (from 1: Never true to 7: Always true), measuring participants' level of cognitive fusion, i.e., one's tendency to get entangled in the content of thoughts. This instrument showed very good psychometric properties in its original ( $\alpha$ s between 0.88 and 0.93 across five samples) and Portuguese ( $\alpha$ s between 0.89 and 0.94 across three samples) studies.

Ruminative Response Scale (RRS-10; [34]; Portuguese version by [35]). The RRS10 measures the level of rumination presented by the participant when feeling sad or with negative mood. It presents 10 items rated on a 4-point scale (0: Almost Never; 3: Almost Always) and two subscales: "reflective rumination" (referring to attempts to comprehend the reasons for negative mood, and to problem resolution) and "brooding" (perseverant thoughts about the negative consequences of negative mood and the obstacles for problem solution). In the present study, only the brooding subscale was used, which showed good psychometric properties in the original $(\alpha=0.77)$ and Portuguese validation studies $(\alpha=$ $0.76)$.

Depression Anxiety Stress Scales (DASS-21 [36]; Portuguese version by [37]). This 21-item instrument assesses the frequency of negative emotional symptoms (involving three subscales: depression, anxiety, and stress) during the week prior to the assessment. Items are measured on a 4-point Likert scale (from 0: "Did not apply to me at all" to 3: "Applied to me very much, or most of the time"); higher scores thus indicate higher psychopathology. Only the depression subscale was considered for the current 
study. This subscale comprises 7 items and has showed good reliabilities in the original $(\alpha=0.88)$ and Portuguese $\left(\alpha_{\mathrm{DEP}}=0.85\right)$ validation studies.

\section{Statistical Analysis}

Data was analysed using SPSS, version 22.0 [38] and IBM AMOS, version 22.0 [39] (Arbuckle, 2013).

The present study aimed to analyse the mediation effects of cognitive fusion (Model 1) and brooding (Model 2) on the association between IBD symptomatology (independent variable - IV) and depressive symptomatology (the outcome). Statistical mediation indicates that the causal effect of an IV on the outcome is explained through the mechanisms of a mediator variable [40]. Longitudinal data allows more mediation analysis options [41, 42], including the cross-lagged panel model for longitudinal data (CLPM; [41]) which has been used in the present study.

Based on structural equation modelling (SEM), the CLPM is a multivariate extension of the univariate simplex model, a structural model commonly used for longitudinal data analysis [43, 44]. In comparison to models using cross-sectional data, the CLPM allows for stronger inference about the direction of causation and reduced probable parameter bias $[41,42]$.

The CLPM involves the assessment of each study variable at multiple times (at least three times to attain a fully longitudinal mediation model), to analyse whether the levels of variables at initial time points impact on values at later time points $[41,45]$. This analysis is done through the examination of the structural associations and the significance of direct and indirect paths (e.g., [46]), through the maximum-likelihood estimation method. In the current study, the bootstrap procedure (with 2000 samples) was used to create $90 \%$ bias-corrected confidence intervals (C.I.s) around the standardized 
estimates of total, direct and indirect effects. If the interval between the lower and the upper bound of the $90 \%$ bias-corrected confidence interval does not comprise zero, the effects are considered statistically significant [47].

The fit of the tested models to the empirical data was assessed through the analysis of several fit indices: chi-square $\left(\chi^{2}\right)$, the Standardized Root Mean Squared Residual (SRMR; which indicates a good model fit when inferior to 0.08; [48]), and the Comparative Fit Index (CFI; that indicates that the model is adequate when above 0.90 and very good when above $0.95 ;[48])$.

Descriptive analyses were conducted using IBM SPSS Statistics [38] and the CLPM was analysed recurring to Amos Software (v. 22; [39]). Specific indirect effects for each model were calculated using AMOS user-defined estimands [49].

\section{Results}

\section{Preliminary analysis}

Skewness and Kurtosis' values indicated that the study variables did not present a significant bias to normal distribution (Skewness varied between 0.01 and 1.56, and Kurtosis between -0.61 and 2.90). Further, visual inspection of distributions corroborated the assumption of normality [47]. Table 1 presents descriptive statistics and the internal consistencies of the study variables.

Please Insert Table 1 around here

Results from correlation analyses can be seen in Table 2. It is interesting to highlight that IBD symptomatology, cognitive fusion, and brooding at baseline were 
positively associated with the experience of depressive symptoms 9 (Wave 2) and 18 months (Wave 3) later.

Please Insert Table 2 around here

\section{Model 1 - The impact of cognitive fusion on depressive symptomatology}

Model 1 (see Figure 1) tested the meditational effect of cognitive fusion on the association between IBD symptomatology at baseline and depression 18 months later This model presented an adequate fit to the empirical data: $\chi 2=52.96, \mathrm{df}=16, p<0.001$; $\mathrm{CFI}=0.95, \mathrm{SRMR}=0.08$, and its effects represented the expected directions.

Please insert Figure 1 around here

IBD symptomatology at Wave 1 presented a significant direct effect on cognitive fusion at Wave $2(\beta=0.098,90 \%$ BCCI $0.016,0.194, p=0.056)$, and in turn cognitive fusion at Wave 2 had a direct effect on depression at Wave 3 ( $\beta=0.427,90 \%$ BCCI 0.269, $0.580, p=0.001)$. Furthermore, IBD symptomatology at Wave 1 presented a specific indirect effect on depression at Wave 3 through the mechanisms of cognitive fusion, which mediated this relationship ( $\beta=0.016,90 \%$ BCCI $0.004,0.039, p=0.028$ ). IBD symptomatology at baseline also impacted on the levels of cognitive fusion 18 months later (Wave 3) through cognitive fusion at Wave $2(\beta=0.08 ; 90 \% \mathrm{BCCI}=0.01$ to $0.16 ; \mathrm{p}$ $=0.05)$.

Furthermore, results also showed that the baseline levels of cognitive fusion predicted depressive symptomatology 18 months later (Wave 3) with an indirect effect of 
$.35(90 \% \mathrm{BCCI}=0.23$ to $0.47 ; p=0.001)$ mediated by the levels of both cognitive fusion and depression at Wave 2 .

Finally, the levels of each variable at baseline predicted the levels of said variable at Wave 3, through the indirect effect of its values on Wave 2. In this way, IBD symptoms at Wave 1 presented an indirect effect of $0.56(90 \% \mathrm{BCCI}=0.43$ to $0.67 ; \mathrm{p}<0.001)$ on symptoms at Wave 3, through the mediator effect of symptomatology at Wave 2. Similarly, cognitive fusion at Wave 1 indirectly impacted on cognitive fusion's levels 18 months later (Wave 3$)$ with an indirect of $.58(90 \% \mathrm{BCCI}=0.48$ to $0.65 ; \mathrm{p}<0.001)$ that was mediated by this process' values at Wave 2. Finally, depressive symptomatology at baseline impacted on depressive severity 18 months later, with an indirect effect of .11 $(90 \% \mathrm{BCCI}=0.02$ to $0.24 ; p<0.05)$ mediated by depression levels at Wave 2 .

The total model accounted for $40 \%$ of depression's variance at wave 3 . Data regarding the model's direct effects are presented in Figure 1 and Table 3.

Please insert Table 3 around here

\section{Model 2 - The impact of brooding on depressive symptomatology}

Model 2 (see Figure 2) analysed brooding's meditational effect on the association between IBD symptomatology at baseline and depression 18 months later. This model presented an adequate fit to the empirical data: $\chi 2=60.97, \mathrm{df}=16, p<0.001 ; \mathrm{CFI}=0.92$, $\mathrm{SRMR}=0.07$. 
Results showed that IBD symptomatology at baseline presented a significant direct effect on brooding 9 months later, at Wave $2(\beta=0.180,90 \%$ BCCI $0.072,0.292$, $p=0.010$ ). In turn, brooding at Wave 2 presented a direct effect on depressive symptoms at Wave $3(\beta=0.210,90 \% \mathrm{BCCI} 0.054,0.345, p=0.038)$. The specific indirect effect between IBD symptomatology at Wave 1 on depressive symptoms at Wave 3, which was totally mediated by brooding, was found significant $(\beta=0.014,90 \% \mathrm{BCCI} 0.004,0.033$, $p=0.017)$. IBD symptomatology at baseline also presented an indirect effect of .12 $(90 \% \mathrm{BCCI}=0.05$ to $0.19 ; p=0.008)$ on brooding's levels 18 months later, through brooding at Wave 2 .

Furthermore, results also showed that brooding at baseline predicted the levels of brooding 18 months later (Wave 3) with an indirect effect of $.46(90 \% \mathrm{BCCI}=0.35$ to $0.56 ; p=0.001)$ mediated by the brooding at Wave 2 . Brooding at baseline also indirectly predicted depressive symptoms at Wave 3 with an effect of $.22(90 \% \mathrm{BCCI}=0.09$ to 0.34 ; $p=0.008)$, through the mechanisms of this emotion regulation process at Wave 2.

The total model accounted for $34 \%$ of depressive symptomatology's variance. Details about the model's direct effects are summarized in Figure 2 and Table 3.

\section{Discussion}

Research has indicated that depressive symptomatology predicts active disease and relapses $[13,14]$ and that this relationship may be due to cellular and humoral immunity alterations caused by depressed mood [15-17]. Furthermore, it is also recognized that IBD activity can increase depressive symptoms, therefore perpetuating a cycle between depression and IBD symptomatology $[19,20]$. It is thus considered particularly relevant to explore the mechanisms that may contribute to the link between IBD symptoms and depressed mood. 
Given that recent research suggests that emotion regulation mediates the impact of adverse experiences on different outcomes [e.g., 22-24], the present study aimed to examine the influence of two maladaptive emotion regulation processes, cognitive fusion and brooding, on the relationship between IBD symptomatology and depressed mood within a period of 18 months. This analysis was conducted with a sample of 116 IBD patients that completed self-report validated measures on an online platform in three different times, equally spaced approximately 9 months apart.

Results from correlation analyses demonstrated that IBD symptomatology, cognitive fusion, and brooding at baseline were positively linked to increased depressed mood 9 and 18 months later. The found association between IBD symptomatology and depressive symptoms has been demonstrated by literature [e.g., 14, 17], whereas this is the first longitudinal study to demonstrate the link of cognitive fusion and brooding with subsequent depressed mood in IBD patients. Depressed mood at baseline was also linked to the experience of depressive symptoms 9 and 18 months later, which indicates that these symptoms appear to be fairly stable over time. Likewise, it is also interesting to note that cognitive fusion at baseline was positively correlated with the level of cognitive fusion manifested 9 and 18 months later. The same was also true for brooding. These results show that these maladaptive emotion regulation strategies may be used by patients in a consistent way, i.e., if a patient engages in these strategies he or she is likely to continue to use them to deal with adverse internal experiences such as sensations, thoughts, memories, or emotions. Moreover, self-reported disease symptomatology also seems to be stable over the time considered in this study, as symptoms at baseline were highly associated with the level of symptomatology at the subsequent times of assessment. 
The main aim of the study was to explore the role of cognitive fusion (model 1) and brooding (model 2) on the association between IBD symptomatology and depressed mood using CLPM, a structural model commonly used for longitudinal data analysis. Both models presented a poor chi-square; nevertheless, given that this index is highly sensible to the complexity of the model, other goodness of fit indices were analysed and the adequacy of both models to the empirical data was confirmed. The models demonstrated that each variable (IBD symptoms, cognitive fusion, brooding, and depressed mood) significantly predicts the levels of that variable at subsequent times (9 and 18 months later). Results also showed that IBD activity significantly predicts subsequent levels of cognitive fusion and brooding, and that, in turn, these processes predict the later experience of depressive symptomatology. Furthermore, cognitive fusion and brooding were found to be significant mediators of the link between IBD symptomatology and subsequent depressed mood.

More specifically, results showed that the experience of IBD symptomatology leads to the engagement in cognitive fusion and brooding. That is, when faced with symptoms, patients may tend to deal with adverse internal experiences (that may comprise the symptoms themselves or related thoughts and emotions) by getting "fused" and ruminative about those experiences, the obstacles for coping with them, and selffocused thoughts. This is a novel finding that makes evident the significant impact of disease activity on patients' psychological processes, namely emotion regulation.

Furthermore, the current study also shows that the tendency to engage in cognitive fusion and brooding to deal with difficult experiences leads to increased subsequent depressive symptoms. This finding supports previous literature concerning the longitudinal effects of brooding on depression levels $[50,51]$ and further expands it by uncovering this causal relationship in IBD patients. On the other hand, to our present 
knowledge, this is the first study to verify the causal predictive value of cognitive fusion on course of depressive symptomatology. The most interesting contribution of this study is, nonetheless, the demonstration that reported IBD symptomatology, although positively associated with subsequent depressed mood, does not directly predict it. This relationship is rather indirect as it is explained by the engagement in cognitive fusion and brooding. These maladaptive emotion regulation processes link the experience of IBD symptoms with the later manifestation of depressed mood.

\section{Limitations}

Some limitations should be considered while interpreting these findings. One limitation might relate to the recruitment process of the sample. Patients were recruited through an Association via an online invitation, which limited the representativeness of the sample by exclusively recruiting patients with access to the internet and registered in the Association. Further, it may be possible that, of the patients invited to participate in this study, those who presented more severe disease activity or more IBD-related complications or concerns were the ones to agree to take part in the study and to complete the three waves of assessment. Another potential limitation of this study is that it relies on self-report measures. Nevertheless, previous studies have used self-reports of disease symptoms to characterize disease activity in IBD samples [e.g., 18]. Future research should collect participants from hospital settings and use larger samples to confirm this study's findings. Moreover, future studies should also use laboratorial medical indices and clinical interviews to assess disease activity and depressive symptomatology, respectively. It would have also been interesting to explore the effects of other emotion regulation processes such as experiential avoidance, uncommitted action, or self- 
judgment, and other outcomes such as social relationships, body image impairment, or sexual functioning.

\section{Conclusions}

The present study is elucidative of the pernicious effects of cognitive fusion and brooding on the link between IBD symptomatology and depressed mood. Given that depressive symptoms greatly impact on patients' well-being, psychosocial functioning, and disease course [13-17], clinicians should be attentive of the emotion regulation patterns used by their patients. High levels of cognitive fusion might be identified by evaluating whether the person excessively believes and gets attached to his or her thoughts, has difficulty considering other perspectives about concerns, fears or difficult experiences, or behaves inflexibly due to dominant painful cognitions. Concerning brooding rumination, clinicians should be aware of indicators of repetitive focus on the obstacles to solve problems and on self-directed thought patterns such as "why did this happen to me?", "what have I done to deserve this?", or "why can't I handle things better?". Patients presenting high levels of these maladaptive processes ought to be referred to psychological assessment.

The implementation of psychological evaluations and interventions in the healthcare of IBD patients should indeed be a main concern. These interventions should focus on diminishing the engagement in cognitive fusion and rumination and promote adaptive emotion regulation strategies such as cognitive defusion, acceptance and mindfulness. Acceptance and Commitment Therapy (ACT) [23], an empirically based psychological intervention, focuses on the promotion of these processes to attenuate the engagement in maladaptive emotion regulation and increase psychological flexibility. Hence, considered the obtained findings, this form of psychotherapy might be especially 
relevant to increase IBD patients' mental health and, potentially, reduce disease activity. The current study may thus serve as an avenue for future research to test the efficacy of ACT-based interventions on IBD sufferers.

\section{Conflict of interest}

The authors declare no conflict of interest.

\section{Acknowledgments}

We thank the Portuguese Association for IBD (Associação Portuguesa para a Doença Inflamatória do Intestino; APDI) for their help during the recruitment process of this study's sample.

\section{Funding}

Research by Inês A. Trindade is supported by a Ph.D. Grant (SFRH/BD/101906/2014) sponsored by FCT (Portuguese Foundation for Science and Technology).

\section{References}

[1] Graff L a., Walker JR., Bernstein CN. Depression and anxiety in inflammatory bowel disease: a review of comorbidity and management. Inflamm Bowel Dis 2009;15(7):1105-18. Doi: 10.1002/ibd.20873.

[2] Janke K-H., Klump B., Gregor M., Meisner C., Haeuser W. Determinants of Life Satisfaction in Inflammatory Bowel Disease. Inflamm Bowel Dis 2005;11(3):27286. Doi: 10.1097/01.MIB.0000160809.38611.f7.

[3] Knowles SR., Gass C., Macrae F. Illness perceptions in IBD influence psychological status, sexual health and satisfaction, body image and relational functioning: A 
preliminary exploration using Structural Equation Modeling. J Crohns Colitis 2013;7(9):e344-50. Doi: 10.1016/j.crohns.2013.01.018.

[4] Canavan C., Abrams KR., Hawthorne B., Drossman D., Mayberry JF. Long-term prognosis in Crohn's disease: factors that affect quality of life. Aliment Pharmacol Ther 2006;23(3):377-85. Doi: 10.1111/j.1365-2036.2006.02753.x.

[5] Casati J., Toner BB., de Rooy EC., Drossman DA., Maunder RG. Concerns of patients with inflammatory bowel disease: a review of emerging themes. Dig Dis Sci 2000;45(1):26-31. Doi: 10.1023/A:1005492806777.

[6] Drossman DA, Ringel Y. Psychological factors in ulcerative colitis and Crohn's disease. In: Sartor R, Sandborn W, Eds. Kirsner's Inflammatory Bowel Disease, 6th edn. Philadelphia, PA, USA: WB Saunders; 2004: 340-356.

[7] Trindade I A, Ferreira C, Pinto-Gouveia J. The effects of body image impairment on the quality of life of non-operated female IBD patients. Qual Life Res 2016. doi: $10.1007 / \mathrm{s} 11136-016-1378-3$

[8] Trindade I A, Ferreira C, Pinto-Gouveia J. Chronic illness-related shame: Development of a new scale and novel approach for IBD patients' depressive symptomatology. Clin Psychol Psychother 2016. doi: 10.1002/cpp.2035

[9] Guthrie E., Jackson J., Shaffer J., Thompson D., Tomenson B., Creed F. Psychological disorder and severity of inflammatory bowel disease predict health-related quality of life in ulcerative colitis and Crohn's disease. Am J Gastroenterol 2002;97(8):1994-9. Doi: 10.1016/S0002-9270(02)04198-9.

[10] Kovács Z., Kovács F. Depressive and anxiety symptoms, dysfunctional attitudes and social aspects in irritable bowel syndrome and inflammatory bowel disease. Int $\mathbf{J}$ Psychiatry Med 2007;37(3):245-55. Doi: 10.2190/PM.37.3.a. 
[11] Walker JR., Ediger JP., Graff LA., Greenfeld JM., Clara I., Lix L., et al. The Manitoba IBD cohort study: A population-based study of the prevalence of lifetime and 12-month anxiety and mood disorders. Am J Gastroenterol 2008;103(8):198997. Doi: 10.1111/j.1572-0241.2008.01980.x.

[12] Ghosh S., Shand A., Ferguson A. Ulcerative colitis. Br Med J 2000;320(7242):111923. Doi: $10.1136 / \mathrm{bmj} .320 .7242 .1119$.

[13] Mardini HE., Kip KE., Wilson JW. Crohn's disease: A two-year prospective study of the association between psychological distress and disease activity. Dig Dis Sci 2004;49(3):492-7. Doi: 10.1023/B:DDAS.0000020509.23162.cc.

[14] Mittermaier C., Dejaco C., Waldhoer T., Oefferlbauer-Ernst A., Miehsler W., Beier M., et al. Impact of depressive mood on relapse in patients with inflammatory bowel disease: a prospective 18-month follow-up study. Psychosom Med 2004;66(1):7984. Doi: 10.1097/01.PSY.0000106907.24881.F2.

[15] Stein M. Stress, depression, and the immune system. J Clin Psychiatry 1989:35-40. Doi: 10.1016/0197-1859(88)90059-3.

[16] Kiecolt-Glaser JK., McGuire L., Robles TF., Glaser R. Psychoneuroimmunology: psychological influences on immune function and health. J Consult Clin Psychol 2002;70(3):537-47. Doi: 10.1037/0022-006X.70.3.537.

[17] Taché Y, Bernstein CN. Evidence for the role of the brain-gut axis in inflammatory bowel disease: Depression as cause and effect? Gastroenterology, 2009;136(7):2058-2061. Doi:10.1053/j.gastro.2009.04.032.

[18] Lix LM., Graff LA., Walker JR., Clara I., Rawsthorne P., Rogala L., et al. Longitudinal study of quality of life and psychological functioning for active, fluctuating, and inactive disease patterns in inflammatory bowel disease. Inflamm Bowel Dis 2008;14(11):1575-84. Doi: 10.1002/ibd.20511. 
[19] Irvine E J. Review article: patients' fears and unmet needs in inflammatory bowel disease. Aliment Pharmacol Ther 2004;20(4):54-59. Doi: 10.1111/j.13652036.2004.02053.x.

[20] Simren M., Axelsson J., Gillberg R., Abrahamsson H., Svedlund J., Bjornsson E. Quality of life in inflammatory bowel disease in remission: the impact of IBS-like symptoms and associated psychological factors. Am J Gastroenterol 2002;97: 389396. Doi: 10.1111/j.1572-0241.2002.05475.x

[21] McCombie AM., Mulder RT., Gearry RB. How IBD patients cope with IBD: A systematic review. J Crohn's Colitis 2013:89-106. Doi: 10.1016/j.crohns.2012.05.021.

[22] Graham CD., Gouick J., Krahé C., Gillanders D. A Systematic Review of the Use of Acceptance and Commitment Therapy (ACT) in Chronic Disease and Long-Term Conditions. Clin Psychol Rev 2016. Doi: 10.1016/j.cpr.2016.04.009.

[23] Hayes SC, Strosahl KD, Wilson KG. Acceptance and commitment therapy: The process and practice of mindful change, 2nd edn. The Guilford Press: New York, 2012.

[24] Segal ZV, Teasdale JD, Williams JMG: Mindfulness-Based Cognitive Therapy Theoretical Rationale and Empirical Status. In Hayes S, Follette V, Linehan MM, Eds. Mindfulness and Acceptance. New York: Guilford Press, 2004:45-65.

[25] Diamond LM., Aspinwall LG. Emotion regulation across the life span: An integrative perspective emphasizing self-regulation, positive affect, and dyadic processes. Motiv Emot 2003:125-56. Doi: 10.1023/A:1024521920068.

[26] Gross JJ. The Emerging Field of Emotion Regulation: An Integrative Review. Rev Gen Psychol 1998;2(3):271-99. Doi: 10.1037/1089-2680.2.3.271. 
[27] Hayes SC., Luoma JB., Bond FW., Masuda A., Lillis J. Acceptance and commitment therapy: model, processes and outcomes. Behav Res Ther 2006;44(1):1-25. Doi: 10.1016/j.brat.2005.06.006.

[28] Gillanders DT., Bolderston H., Bond FW., Dempster M., Flaxman PE., Campbell L., et al. The Development and Initial Validation of the Cognitive Fusion Questionnaire. Behav Ther 2014;45(1):83-101. Doi: 10.1016/j.beth.2013.09.001.

[29] Trindade IA., Ferreira C., Pinto-Gouveia J. Ulcerative Colitis Symptomatology and Depression: The Exacerbator Role of Maladaptive Psychological Processes. Dig Dis Sci 2015;60(12):3756-63. Doi: 10.1007/s10620-015-3786-6.

[30] Luoma JB, Hayes SC. Cognitive defusion. In: O’Donahue W, Fisher JE, Hayes SC, Eds. Empirically supported techniques of cognitive behavioral therapy: a step-bystep guide for clinicians, 2nd ed. New York: Wiley, 2003: 71-78.

[31] Morrow J., Nolen Hoeksema, S. Effects of responses to depression on the remediation of depressive affect. J Pers Soc Psychol 1990;58(3):519-527. Doi: $10.1037 / 0022-3514.58 .3 .519$

[32] Nolen-Hoeksema S. The role of rumination in depressive disorders and mixed anxiety/depressive symptoms. J Abnorm Psychol 2000;109(3):504-11. Doi: 10.1037/0021-843X.109.3.504.

[33] Nolen-Hoeksema S., Wisco BE., Lyubomirsky S. Rethinking Rumination. Perspect Psychol Sci 2008;3(5):400-24. Doi: 10.1111/j.1745-6924.2008.00088.x.

[34] Treynor W., Gonzalez R., Nolen-Hoeksema S. Rumination reconsidered: A psychometric analysis. Cognit Ther Res 2003;27(3):247-59. Doi: 10.1023/A:1023910315561. 
[35] Dinis A., Gouveia JP., Duarte C., Castro T. Estudo de validação da versão portuguesa da Escala de Respostas Ruminativas [Study of the Portuguese validation of the Ruminative Responses Scale]. Psychologica 2011;54:175-202.

[36] Lovibond S H., Lovibond P F. Manual for the Depression Anxiety Stress Scales, 2 edn. Sydney: Psychology Foundation, 1995.

[37] Pais-Ribeiro JL., Honrado A., Leal I. Contribuição para o estudo Da adaptação portuguesa das escalas de ansiedade, depressão e stress (EADS) de 21 Itens de Lovibond e Lovibond [Contribution for the study of the Portuguese adaptation of the 21-item Lovibond and Lovibond's Depression Anxiety and Stress Scales]. Psicol Saúde Doenças 2004;5(2):229-239.

[38] IBM Corp. Released 2013. IBM SPSS Statistics for Windows, Version 22.0 [Computer software]. Armonk, NY: IBM Corp.

[39] Arbuckle, J. L. (2013). Amos 22.0 User's Guide. Chicago: IBM SPSS.

[40] Hoyle R H, Kenny D A. Sample size, reliability, and tests of statistical mediation. In Hoyle R H, Ed., Statistical strategies for small sample research. Thousand Oaks, CA: Sage, 1999: 195-222

[41] Cole D A, Maxwell SE. Testing mediational models with longitudinal data: questions and tips in the use of structural equation modeling. $\mathrm{J}$ Abnorm Psychol 2003;112(4):558-77. Doi: 10.1037/0021-843X.112.4.558.

[42] MacKinnon D P. Longitudinal mediation analysis. In MacKinnon D P, Ed.. Introduction to statistical mediation analysis. New York: Lawrence Erlbaum, 2008: $193-236$.

[43] Jöreskog KG. Estimation and testing of simplex models. Br J Math Stat Psychol 1970;23(2):121-45. Doi: 10.1002/j.2333-8504.1970.tb00599.x. 
[44] Jöreskog KG. Statistical estimation of structural models in longitudinal developmental investigations. In Nesselroade J R, Baltes P B, Eds. Longitudinal research in the study of behavior and development. New York: Academic Press, 1979: 129-169.

[45] Selig JP., Preacher KJ. Mediation models for longitudinal data in developmental research. Res Hum Dev 2009;6(2-3):144-64. Doi: 10.1080/15427600902911247.

[46] Schumacker R E, Lomax R G A beginner's guide to structural equation modeling. Mahwah, NJ: Lawrence Erlbaum Associates, 2004.

[47] Kline R B. Principle and practice of structural equation modeling. New York: Guilford, 2005.

[48] Hu L., Bentler PM. Cutoff criteria for fit indexes in covariance structure analysis: Conventional criteria versus new alternatives. Struct Equ Model A Multidiscip J 1999;6(1):1-55. Doi: 10.1080/10705519909540118.

[49] Amos development corporation (2010). User-defined estimand: Multiple estimands. http://www.amosdevelopment.com/video/user-defined-estimands/multipleestimands/amos20/multiple-estimands-amos20.html

[50] Cox S, Funasaki K, Smith L, Mezulis AH. A prospective study of brooding and reflection as moderators of the relationship between stress and depressive symptoms in adolescence. Cognit Ther Res 2012;36(4):290-9. Doi: 10.1007/s10608-0119373-z.

[51] Jose PE, Weir KF. How is Anxiety Involved in the Longitudinal Relationship between Brooding Rumination and Depressive Symptoms in Adolescents? J Youth Adolesc 2013;42(8):1210-22. Doi: 10.1007/s10964-012-9891-3. 
Table 1. Descriptive statistics and Cronbach's alphas $(N=116)$

\begin{tabular}{|c|c|c|c|c|}
\hline \multirow{2}{*}{\multicolumn{2}{|c|}{ Demographic and medical data at Wave 1}} & $n$ & \multirow[t]{2}{*}{$M$} & \multirow[t]{2}{*}{$S D$} \\
\hline & & & & \\
\hline \multirow{2}{*}{ Gender } & Male & $35(30.17 \%)$ & & \\
\hline & Female & $81(69.83 \%)$ & & \\
\hline \multicolumn{2}{|l|}{ Age (range: 18-75) } & & 36.76 & 11.39 \\
\hline \multicolumn{2}{|c|}{ Education (range: $7^{\text {th }}$ grade $-\mathrm{PhD}$ ) } & & 14.75 & 2.74 \\
\hline \multirow{4}{*}{ Marital status } & Single & $54(46.55 \%)$ & & \\
\hline & Married or Cohabitating & $54(46.55 \%)$ & & \\
\hline & Divorced & $7(6.03 \%)$ & & \\
\hline & Widowed & $1(0.86 \%)$ & & \\
\hline \multirow{3}{*}{ Type of IBD } & Crohn's Disease & $70(60.34 \%)$ & & \\
\hline & Ulcerative Colitis & $43(37.07 \%)$ & & \\
\hline & IBD-unknown & $3(2.59 \%)$ & & \\
\hline \multirow{4}{*}{$\begin{array}{l}\text { Time since } \\
\text { diagnosis }\end{array}$} & Until a year & $5(4.31 \%)$ & \multirow{4}{*}{8.76} & \multirow{4}{*}{6.93} \\
\hline & $1-5$ years & $40(34.48 \%)$ & & \\
\hline & $6-10$ years & $35(30.17 \%)$ & & \\
\hline & $>10$ years & $36(31.03 \%)$ & & \\
\hline \multirow{5}{*}{$\begin{array}{c}\text { Most reported } \\
\text { associated medical } \\
\text { complications }\end{array}$} & osteoarticular complaints & $13(11.21 \%)$ & & \\
\hline & dermatological complaints & $11(9.48 \%)$ & & \\
\hline & anorectal pathology & $10(8.62 \%)$ & & \\
\hline & anaemia & $3(2.59 \%)$ & & \\
\hline & hepatic problems & $3(2.59 \%)$ & & \\
\hline \multirow{4}{*}{ Medication usage } & $\begin{array}{l}\text { 5-aminosalicylic acid (5- } \\
\text { ASA) therapies }\end{array}$ & $50(43.10 \%)$ & & \\
\hline & Corticosteroids & $22(18.97 \%)$ & & \\
\hline & Immunomodulators & $63(54.31 \%)$ & & \\
\hline & Biologics & $35(30.17 \%)$ & & \\
\hline
\end{tabular}




\begin{tabular}{|c|c|c|c|c|}
\hline \multirow{3}{*}{$\begin{array}{l}\text { Number of } \\
\text { surgeries }\end{array}$} & 0 & $84(72.41 \%)$ & \multirow{3}{*}{1.97} & \multirow{3}{*}{1.80} \\
\hline & 1 & $19(16.38 \%)$ & & \\
\hline & $>1$ & $13(11.21 \%)$ & & \\
\hline
\end{tabular}




\section{Table 2}

Means (M), Standard Deviations (SD), Cronbach's alphas ( $\alpha$ ) and intercorrelation scores of the study variables in the three waves $(N=116)$

\begin{tabular}{|c|c|c|c|c|c|c|c|c|c|c|c|c|c|c|}
\hline & $M$ & $S D$ & $\alpha$ & 1 & 2 & 3 & 4 & 5 & 6 & 7 & 8 & 9 & 10 & 11 \\
\hline 1. IBD symptoms at $\mathrm{W} 1$ & 21.48 & 10.03 & 0.85 & ـ & & & & & & & & & & \\
\hline 2. Cognitive Fusion at W1 & 22.76 & 10.39 & 0.95 & 0.14 & & & & & & & & & & \\
\hline 3. Brooding at $\mathrm{W} 1$ & 6.11 & 3.43 & 0.83 & 0.14 & $0.68^{* * *}$ & - & & & & & & & & \\
\hline 4. Depressive symptoms at $\mathrm{W} 1$ & 4.12 & 4.26 & 0.90 & $0.32 * *$ & $0.64 * * *$ & $0.56 * * *$ & - & & & & & & & \\
\hline 5. IBD symptoms at $\mathrm{W} 2$ & 20.41 & 9.63 & 0.84 & $0.71 * * *$ & 0.13 & 0.11 & $0.25 * *$ & - & & & & & & \\
\hline 6. Cognitive Fusion at W2 & 20.94 & 10.09 & 0.96 & $0.27 * *$ & $0.73^{* * * *}$ & $0.57 * * *$ & $0.58^{* * *}$ & $0.24 * *$ & - & & & & & \\
\hline 7. Brooding at $\mathrm{W} 2$ & 5.56 & 3.10 & 0.81 & $0.30^{* *}$ & $0.63^{* * *}$ & $0.72^{* * * *}$ & $0.53^{* * * *}$ & 0.16 & $0.72 * * *$ & - & & & & \\
\hline 8. Depressive symptoms at W2 & 3.67 & 3.10 & 0.90 & $0.31 * *$ & $0.46^{* * *}$ & $0.42 * * *$ & $0.60 * * *$ & $0.32 * *$ & $0.71 * * *$ & $0.52 * * *$ & - & & & \\
\hline 9. IBD symptoms at W3 & 20.34 & 10.01 & 0.86 & $0.74 * *$ & 0.13 & 0.06 & $0.25^{* *}$ & $0.80 * * *$ & $0.24 * *$ & 0.15 & $0.25^{* *}$ & - & & \\
\hline 10. Cognitive Fusion at W3 & 21.24 & 10.82 & 0.97 & $0.24 *$ & $0.63^{* * *}$ & $0.57 * * *$ & $0.48^{* * *}$ & 0.12 & $0.80^{* * * *}$ & $0.68 * * *$ & $0.61^{* * *}$ & $0.20^{*}$ & - & \\
\hline 11. Brooding at $\mathrm{W} 3$ & 5.40 & 3.34 & 0.83 & $0.25^{* *}$ & $0.51^{* * *}$ & $0.63 * * *$ & $0.39 * * *$ & $0.21^{*}$ & $0.61 * * *$ & $0.66^{* * *}$ & $0.49^{* * *}$ & $0.25^{* *}$ & $0.64 * * *$ & - \\
\hline 12. Depressive symptoms at W3 & 3.78 & 3.90 & 0.89 & $0.25^{* *}$ & $0.51^{* * *}$ & $0.40^{* * *}$ & $0.59^{* * *}$ & $0.26 * *$ & $0.61^{* * *}$ & $0.45^{* * *}$ & $0.59^{* * *}$ & $0.27^{* *}$ & $0.70^{* * *}$ & $\begin{array}{c}0.49^{* *} \\
*\end{array}$ \\
\hline
\end{tabular}

Note: ${ }^{*} p<0.05 ;{ }^{* *} p<0.01 ;{ }^{* * *} p<0.001$.

$\mathrm{W} 1=$ Wave $1 ; \mathrm{W} 2=$ Wave $2 ; \mathrm{W} 3=$ Wave 3 
Table 3. Unstandardized path coefficients of the direct associations for the analysed longitudinal mediation models

\begin{tabular}{|c|c|c|}
\hline & Model 1 & Model 2 \\
\hline & Estimate (SE) & Estimate (SE) \\
\hline 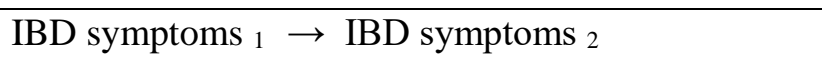 & $0.82(0.08)^{* * *}$ & $0.82(0.08)^{* * *}$ \\
\hline IBD symptoms $_{2} \rightarrow$ IBD symptoms $_{3}$ & $0.85(0.06)^{* * *}$ & $0.82(0.06)^{* * *}$ \\
\hline 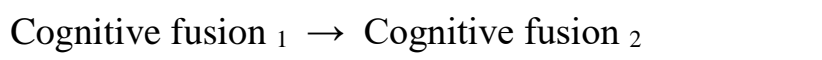 & $0.69(0.06)^{* * *}$ & - \\
\hline 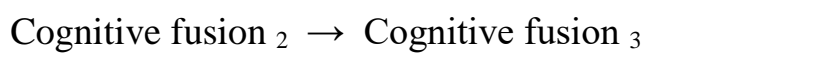 & $0.85(0.06)^{* * *}$ & - \\
\hline Brooding $_{1} \rightarrow$ Brooding $_{2}$ & - & $0.63(0.06)^{* * *}$ \\
\hline Brooding $_{2} \rightarrow$ Brooding $_{3}$ & - & $0.70(0.08)^{* * *}$ \\
\hline Depressive symptoms $_{1} \rightarrow$ Depressive symptoms $_{2}$ & $0.38(0.07)^{* * *}$ & $0.45(0.08)^{* * *}$ \\
\hline Depressive symptoms $_{2} \rightarrow$ Depressive symptoms $_{3}$ & $0.27(0.09)^{* *}$ & $0.45(0.08)^{* * *}$ \\
\hline IBD symptoms $_{1} \rightarrow$ Cognitive Fusion $_{2}$ & $0.09(0.05)$ & - \\
\hline IBD symptoms $_{1} \rightarrow$ Brooding $_{2}$ & - & $0.06(0.02)^{* *}$ \\
\hline Cognitive Fusion $_{1} \rightarrow$ Depressive symptoms $_{2}$ & $0.07(0.03)^{*}$ & - \\
\hline Brooding $_{1} \rightarrow$ Depressive symptoms $_{2}$ & - & $0.16(0.10)$ \\
\hline IBD symptoms $_{1} \rightarrow$ Depressive symptoms $_{3}$ & & $0.01(0.03)$ \\
\hline Cognitive Fusion $_{2} \rightarrow$ Depressive symptoms $_{3}$ & $0.16(0.04) * * *$ & - \\
\hline Brooding $_{2} \rightarrow$ Depressive symptoms $_{3}$ & - & $0.27(0.11)^{*}$ \\
\hline
\end{tabular}

Note: ${ }^{*} p<0.05 ; * * p<0.01 ; * * * p<0.001$ 
Wave 1

Baseline
Wave 2

9 months
Wave 3

18 months

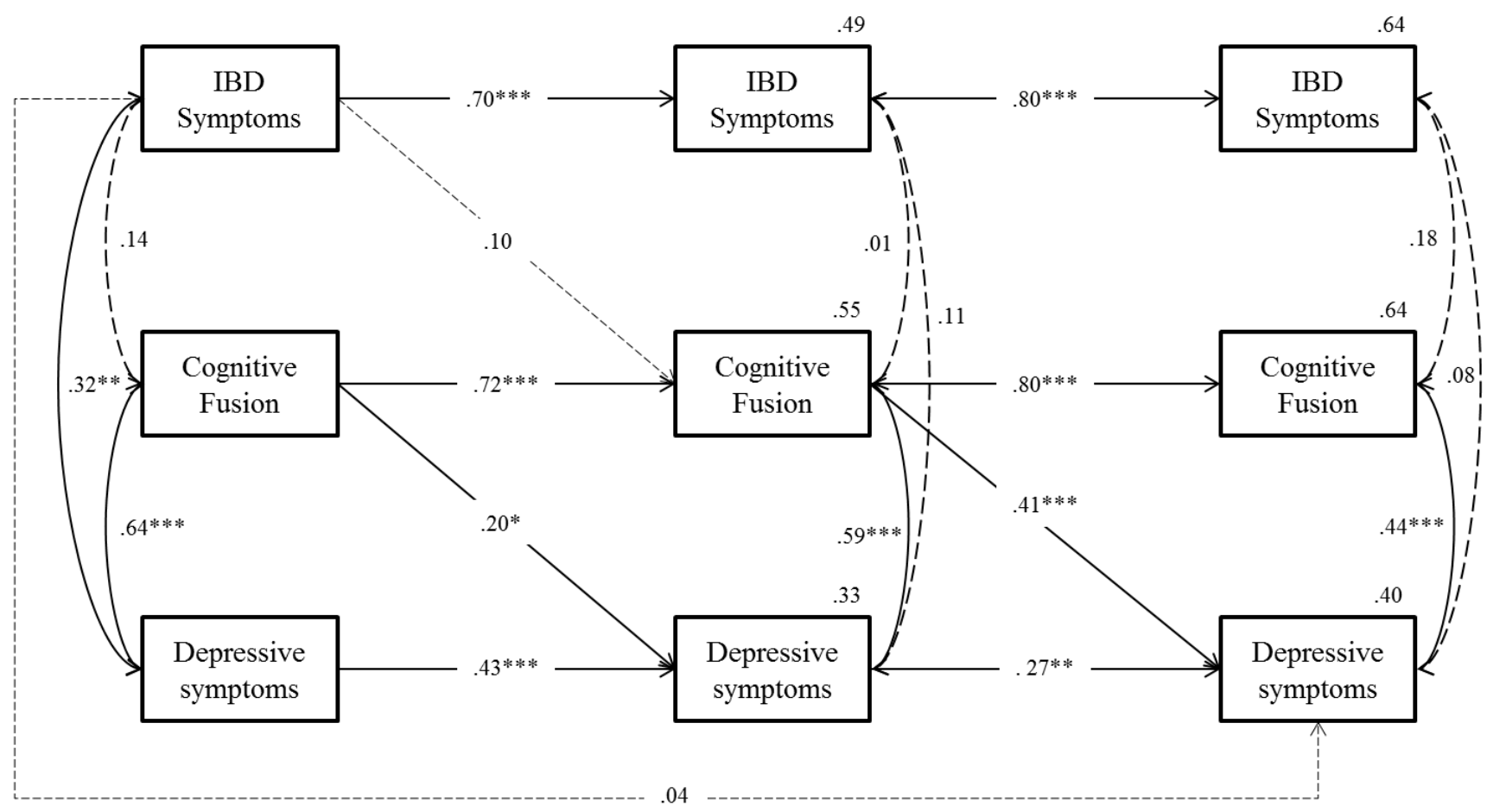

Figure 1.

Note. Standardized path coefficients among variables are presented.

${ }^{*} p<0.05 ;{ }^{* *} p<0.01 ;{ }^{* * *} p<0.001$. 
Wave 1

Baseline
Wave 2

9 months
Wave 3

18 months

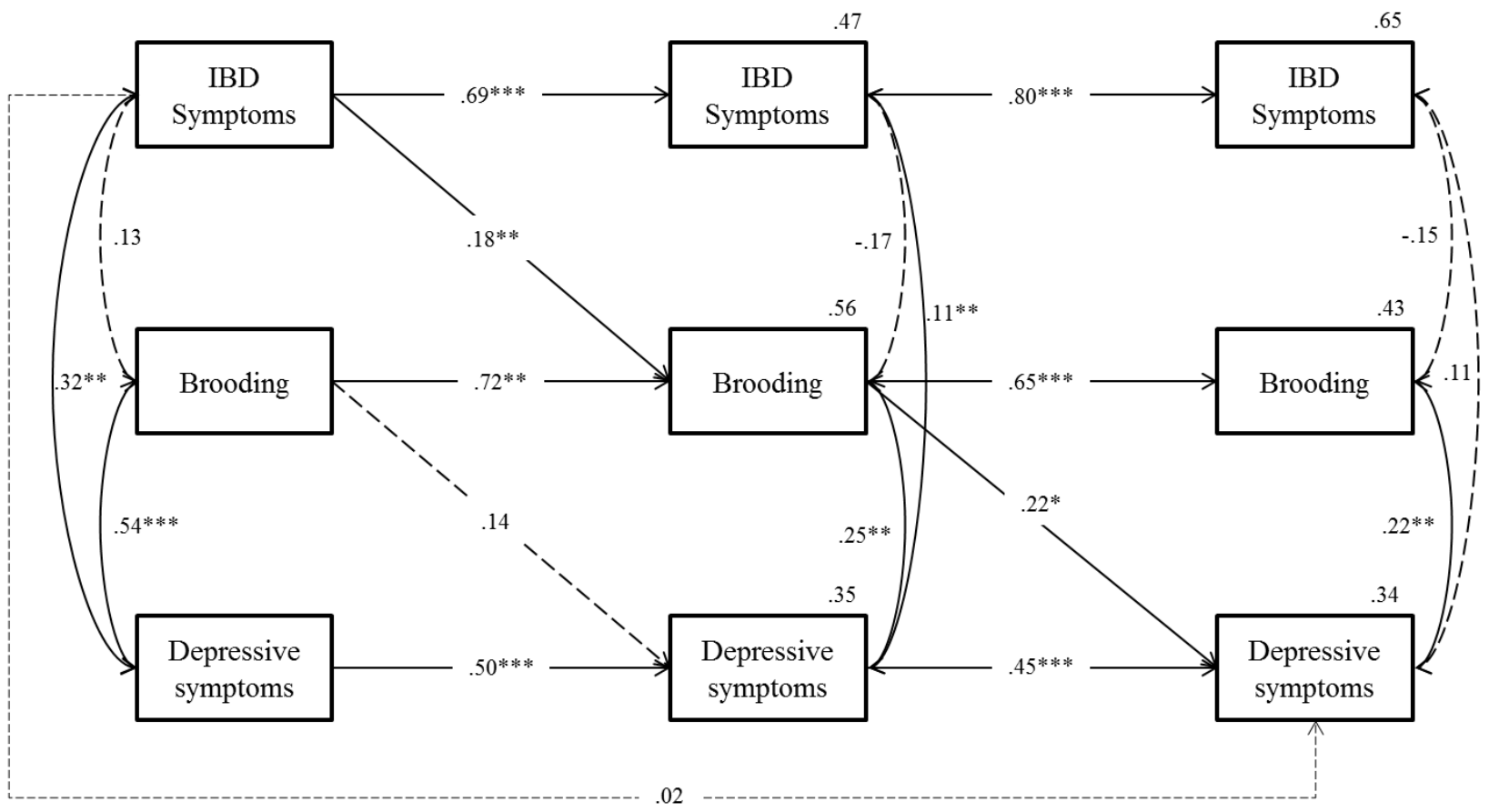

Figure 2.

Note. Standardized path coefficients among variables are presented.

${ }^{*} p<0.05 ;{ }^{* *} p<0.01 ;{ }^{* * *} p<0.001$. 